\title{
Intervention Programme Based on Executive Functions in Children with ADHD
}

\author{
María Jesús Cardoso-Moreno ${ }^{\dagger}$, Santos Orejudo Hernandez, Cesar Rodriguez Ledo
}

\begin{abstract}
Attention-deficit-hyperactivity disorder (ADHD) is a common neurobehavioral disorder with onset in childhood. Children with ADHD have been found to show impairment in emotional self-control, self-regulation and motivation. Numerous studies have reported cognitive disabilities in memory, executive functions, spatial abilities and language skills. The main objective of this research was to determine if the executive function intervention programme implemented could improve attention process and executive functions in children with Attention Deficit Hyperactivity Disorder. The sample consisted of 50 children (14 female and 36 male) aged between 8 and 12 years, diagnosed with ADHD and who were not under any psychopharmacological treatment at the time of the study, and had not taken medication to mitigate such disorder. Executive functioning was assessed through the Zoo Map Test and attention was evaluated by the "D2 Test of Attention" in a pretest-postest cuasi experimental design. The executive functioning intervention programme implemented was designed and tested for this research and consisted of 12 one-hour weekly sessions and the participants were trained individually. The data indicated that the intervention lead to improved performance in the execution of tasks that evaluate executive functions. After the intervention, the children took less time to resolve the Zoo Map Test, and the results in the "D2 Test of Attention" also improved after the training. Therefore, data showed that such executive functioning intervention programme could improve attention processes and executive functions in children with Attention Deficit Hyperactivity Disorder. Therefore, the programme used becomes effective intervention as well as the data found links the nature of the problem to a specific treatment management by objectives.
\end{abstract}

\section{Keywords}

Attention deficit hyperactive disorder, Intervention programme, Executive functions, D2, Attention, Zoo Map Test, Gender, Child interventions

\begin{abstract}
Introduction
Attention deficit hyperactivity disorder (ADHD), a common neurobehavioral disorder with onset in childhood [1], is characterized by developmentally inappropriate levels of hyperactivity, motor impulsivity, emotional and social responses, a general lack of inhibition and pervasive inattention. In many studies, $\mathrm{ADHD}$ has been conceptualized as resulting from dysfunction
\end{abstract}

in the frontostriatal region of the prefrontal cortex and manifesting as observable deficits in higherorder executive function. This conceptualization of $\mathrm{ADHD}$ presumes that the essential impairment in $\mathrm{ADHD}$ is a deficit in behavioural response inhibition leading to secondary impairments in Executive Functions (EF).

The core symptoms of inattention, impulsivity, and motor activity are found to be highly 
correlated with neuroanatomical differences and so Stuss and Alexander [2] have been identified discrete categories of function of the frontal lobes. These researchers indicated, however, that the executive category does not refer solely to a central executive system and that the frontal lobes do not have a unitary organizing role, but rather described impairments in a multitude of anatomical and functional attentional control processes that are interrelated. This argument supports the view that the frontal lobes work in tandem with many other areas of the brain.

Executive function has been said to have many dimensions, including choosing, planning, programming, implementing, monitoring, and adjusting or ending a volitional act. Attention can be considered as another facet of EF and, therefore, can be selective and well sustained when functioning appropriately. On the other hand, attention can be discriminating and, in some ways, biased toward achieving an end or desired goal [3].

Moreover, several authors have proposed that symptoms of ADHD arise from a primary deficit in a specific EF domain such as response inhibition or working memory or a more general weakness in executive control [4-8]. This hypothesis is based on the observation that prefrontal lesions may produce behavioural hyperactivity, distractibility, or impulsivity, as well as deficits on EF tasks [9,10]. In a multifactorial neuropsychological model of ADHD, EF weaknesses may be one of several important components.

Neuroimaging functional studies in children diagnosed with $\mathrm{ADHD}$ have shown consistent abnormalities relative to control subjects in late-developing inferior frontostriatal and frontocerebellar circuitries. Such circuits have been said to mediate the cognitive control functions that are impaired in the disorder. In this sense, structural magnetic resonance imaging (MRI) studies found reduced volume and cortical thickness in inferior prefrontal cortex (IFC) but also other frontal brain regions, as well as parietotemporal regions, the basal ganglia, the splenium of the corpus callosum, and the cerebellum [11-13]. In the same direction, Chafee and Goldman-Rakic [14] have emphasized the role of a frontoparietal system in visual attention/working memory tasks and showed the role and anatomy of the dopaminergic components of this circuit.
In relation to structure, longitudinal imaging studies have provided evidence about how the structural abnormalities in these late-developing fronto-striato-cerebellar and frontoparietal systems are due to a late structural maturation of these regions [5]. Thus, the peak of cortical thickness maturation has been shown to be delayed in children with ADHD compared with healthy peers by an average of 3 years, with some regions, including frontal and temporal areas, being delayed in their cortical maturation by up to 4 to 5 years [15].

In relation to circuits, several studies conclude that dorsal frontostriatal, orbitofronto-striatal, and fronto-cerebellar circuits are involved in ADHD. These circuits seem to interact through spiralling loops in the striatum and connections from the cerebellum to the prefrontal cortex and the striatum, although there are more connections within such structures than between circuits. Dysfunction in any of these circuits might cause symptoms of ADHD. According to Casey et al. [16] findings, dysfunction of the prefrontal cortex is likely to result in a reduced ability to exert control. They dysfunction in dorsal striatum might lead to differences in the ability to predict what events are going to occur subsequently, whereas dysfunction in ventral striatum is more likely to lead to deficits in motivation and reward processing. Moreover, dysfunction of the cerebellum is likely associated with problems in the ability to predict when events are going to occur and other problems with timing. The implication of this is that, although this wide range of neurobiological differences can lead to symptoms of ADHD, the cognitive effects of dysfunction at the various levels might be quite different. Preliminary evidence that such dissociation might be possible comes from Sonuga-Barke et al. [17] who used principal component analysis to show that three separable components contributed to the variance in their neuropsychological task battery. These components corresponded to timing, cognitive control or inhibition, and reward or delay aversion. Our of the 77 children with ADHD included in this study, 55 could be identified as having a deficit on one of these components, and the overlap between components was no greater than would be expected by chance [17]. This finding brought the authors to suggest that these components might indeed rely on neurobiological separable systems. The cognitive areas with which these components are related suggest they might map onto the three circuits described: timing is 
associated with fronto-cerebellar loops, cognitive control with dorsal frontostriatal loops, and reward with orbitofronto-striatal loops.

There might be at least three neurobiological pathways to ADHD, involving disruption of dorsal frontostriatal, orbitofronto-striatal, or fronto-cerebellar circuits. However, there might be other circuits involved. In this sense, the amygdala has been found to have reciprocal connections with the prefrontal cortex and has been suggested to play a role in ADHD [18]. According to Nigg and Casey [18] findings fronto-amygdalar circuits are involved in attributing emotional value to events and inputs from amygdala could affect the recruitment of prefrontal control. On the other hand, only indirect evidence of the involvement of this circuitry in ADHD comes from the observation of differences in medial temporal lobe structures in ADHD [19-21]. In relation to such findings and based on the three circuits that evidence suggest could be damaged in ADHD: dorsal frontostriatal, orbitofronto-striatal, and frontocerebellar; the aim of this study was to test whether the executive functioning programme implemented could improve the attention process in children with ADHD.

\section{Methods}

\section{- Participants}

The sample of this study consisted of 50 children, 14 female and 36 male, aged between 8 and 12 years, diagnosed with ADHD-predominantly hyperactive/impulsive presentation, ac $(\mathrm{X}=10.24 ; \mathrm{SD}=3.56)$ according to DSM-5 criteria. They were not under any psychopharmacological treatment at the time of the study, and had not taken medication to treat such ADHD previously. The participants were students from various schools from the province of Zaragoza, Spain. All participants, and their parents, received information about the purpose of the intervention and agreed to participate voluntarily. Subjects received no compensation for participating in the study. Compliance with the standards contained in the Declaration of Helsinki on human experimentation was guaranteed at all times. Inclusion criteria included being aged between 8 and 12, attending any school in the province of Zaragoza (Spain) and having been diagnosed with ADHD Exclusion criteria included presenting some kind of mental disorder, intellectual disability or visual/hearing impairment. Students who were taking, or had been taking, psychopharmacological treatment for ADHD and those students who had previously attended psychological therapy were also excluded.

\section{- Instruments and design}

As dependent variable, selective and sustained attention were assessed through the D2 Test of Attention [22] in a pretest-postest design study. The D2 Test of Attention is a neuropsychological measure of such types of attention, in which children must quickly cross out all d's with 2 marks of any kind. Each line is present for $20 s$ and then the respondent is asked to move to the next one.

The other dependent variable studied was executive functioning, which was assessed through the Zoo Map test (version A in pre-test phase and $B$ in post-test phase in order to avoid any memory effect) [23]. The Zoo Map Test is one of the subtests of behavioural assessment of Dysexecutive syndrome (BADS), a battery of tests aimed at predicting the difficulties in daily life as a result of Dysexecutive syndrome. As independent variable, a training of an executive function intervention was implemented. The training consisted of 12 one-hour weekly sessions and it was carried out individually, according to the intervention programme designed to improve such attention dimensions.

\section{- Procedure}

Before initiating the study, parents were interviewed and informed about the programme interventions, its goal and the sessions to be carried to achieve it, as well as the research which would be carried out. Afterwards, they were asked if they were willing to participate in the study and given an informed consent for their children to be participants of it. Then, and before starting the intervention we administrated the D2 Test of Attention and the Zoo Map Test. The participants then initiated the sessions of the intervention programme which were implemented individually and inside a soundproof room adapted for children. Each of the sessions was started with a relaxation activity focused on breathing. Then, the participants were informed about the rules that had to be followed during the exercises: "I have to pay attention to the explanation of the activity", then "I have to do the task," "I have to check what I have done" and finally, "I congratulate myself for my work". Later on, the participants were trained to develop the capacity to plan with mazes that differ in complexity and activities where they had to follow instructions and 
rules carefully. Finally, we worked on how we can change our emotions according with the cognitive-behavioural approach, according to the design of the programme. To this matter, the association between emotion and thought was addressed and the participants worked on substituting negative emotions with positive ones through our thoughts.

Once this part of the study was finished, the D2 Test of Attention and Zoo Map Test were administrated again.

\section{- Data analysis}

Data was analysed using IBSM SPSS 20.0 for Windows. The statistical techniques were on the hand descriptive, and so arithmetic mean, standard deviation and effect size were analysed. On the other hand, group comparative for a related sample using the T-test analysis were also carried out to measure the pre-postest differences. Such differences were deemed significant when $\alpha<.05$.

\section{Results}

The results found in both test before and after the implementation of the executive function programme intervention are showed at the following table (Table 1).

The data informs about an improvement in D2 test $(\mathrm{t}=6.51 ; \mathrm{p}=0.001)$ and Zoo Map Test $(t=6.56 ; p=0.000)$ after the executive function programme intervention.

According to gender, the analysis of the D2 scores we found statistical differences in pretest $(\mathrm{t}=2.54 ; \mathrm{p}=0.038)$ and in postest $(\mathrm{t}=2.57$; $\mathrm{p}=0.037$ ), being the improvement in D2 Scores better in girls than boys (Table 2). No statistically significant differences were found with regards to sex in the Zoo Map Test.

Table 1: Pretest-Post test data in D2 Test of Attention and Zoo Map Test.

\begin{tabular}{|l|l|l|l|l|}
\hline Test & Mean (S.D.) & t & p & Cohen's d \\
\hline $\begin{array}{l}\text { D2 Attention Test } \\
\text { Pretest }\end{array}$ & $92.6(48.8)$ & & & \\
\hline Post test & $192.8(70.5)$ & 6.51 & .001 & 1.65 \\
\hline Zoo Map Test & & & & \\
\hline Pretest & $3.4(1.3)$ & 6.56 & 0.000 & 0.51 \\
\hline Post test & $2.4(2.4)$ & & & \\
\hline
\end{tabular}

Table 2: Gender differences Pretest-Post test data in D2 Test of Attention.

\begin{tabular}{|c|c|c|c|c|c|c|c|}
\hline \multirow[b]{2}{*}{ D2 Scores } & \multicolumn{2}{|l|}{ Boys } & \multicolumn{2}{|l|}{ Girls } & \multirow[b]{2}{*}{$\mathbf{t}$} & \multirow[b]{2}{*}{$\mathbf{p}$} & \multirow[b]{2}{*}{ Cohen's o } \\
\hline & M & SD & M & SD & & & \\
\hline Pretest & 102.8 & 47.08 & 47.08 & 31.02 & 2.54 & .038 & 1.52 \\
\hline Postest & 207.6 & 67.77 & 119.0 & 30.30 & 2.57 & .037 & 1.68 \\
\hline
\end{tabular}

\section{Discussion}

According to previous studies, executive function deficits are suggested to be an important component of attention-deficit hyperactivity disorder (ADHD) [24]. In many studies ADHD has been associated with weaknesses in several key EF domains. EF difficulties appear to be one of several important weaknesses that compromise the overall neuropsychological ethology of ADHD [25,26]. Therefore, the main aim of this study was to determine if the executive function intervention programme designed would improve attention deficits and executive functioning. Previous literature indicate that this type of interventions can improve performance in attention tasks and executive functioning and so this paper assess how this specific intervention succeeds on achieving such goal.

Childhood ADHD is said to be characterized by structural and functional deficits compared to healthy children. This may occur in predominantly inferior but also medial and dorsolateral prefrontal cortices, anterior cingulate, the basal ganglia, cerebellum, and temporo-parietal brain regions and their functional and structural interconnectivity, causing poor top-down control over inhibitory, attention, and timing functions. Moreover, it has been suggested that neurobiological dysfunction in any of these circuits can lead to symptoms of ADHD. Deficits in the prefrontal cortex could affect control systems directly, as well as problems in the circuits relaying information to the prefrontal cortex could lead to reduced signalling for control [16]. In both scenarios, behavioural control would be compromised, leading to behavioural changes, such as impulsive and inattentive behaviour. This data has been taken into account for the designing of our intervention programme, and so strategies that address executive function processes, therefore, provide a starting point for improving academic performance. When students use executive function strategies, it is expected that they may become more efficient and begin to improve academically. To build their motivation and persistence, students may need to understand their strengths and weaknesses, which strategies work well for them, as well as why, where, when, and how to apply specific strategies. This understanding referred to as metacognition, or the ability to think about their own thinking and learning, underlies students' use of executive function processes. Students need to learn when to use which 


\section{Intervention Programme Based on Executive Functions in Children with ADHD Research}

strategies and in what contexts. They also need to recognize that not all strategies work for all tasks and all content areas. In other words, strategies need to fit well with the student's learning style as well as the task content and the context. The data of our study shows that the intervention programme implemented improves attention process and executive function strategies in the participants. Students' attention and their ability to engage actively in the learning process are often associated with their ability to regulate their emotions in and outside the classroom [27-29]. The effects of emotion on the learning process range along a continuum. Specifically, extreme emotional reactions (e.g., anxiety, anger) often disrupt students' attention and ability to stay on task as well as their ability to learn and remember new information [30,28]. In contrast, moderate emotional arousal has a positive influence on students' attention and executive function processes including working memory, cognitive flexibility, and inhibition [31,28]. More specifically, the relationship between anxiety and performance is characterized by an inverted U-shaped curve, e.g., test performance is often poor when students either are not anxious and have consequently not studied or are excessively anxious which interferes with attention, working memory, and overall performance [32]. Similarly, negative moods disrupt attention, concentration, memory and processing speed. Our results show that including emotional aspects in intervention programs may improve executive functioning and attention process. This data is in accordance with other studies.

In relation to the intervention itself, information about the child's neuropsychological, cognitive, academic, and psychosocial status forms the basis for designing integrated intervention and treatment plans for children and adolescents with brain-related disorders. Efforts to develop models of neuropsychological intervention have been expanding in recent years and so, in an effort to provide a framework for linking assessment to interventions, the Amsterdam attention and memory training programme has been designed according to a modified version of the Sohlberg and Mateer model [33]. This programme contains elements that address process training for attention and memory, metacognitive strategy training, social contact and support. Hooft et al. [34] found that, following an executive intervention treatment, children showed an improvement on neuropsychological measures of sustained and selective attention; a modest improvement on memory tests, and improvement on teacher and parent reports of behaviours, learning and social-emotional factors. Other interventions that promote executive control skills have revealed to improve behaviour in children with ADHD [35].

According to gender, being male or female has also been considered an important variable in ADHD research. However, many findings, such as Gomez [36], do not find any significant gender differences in children diagnosed with ADHD. Our results coincide with this findings, since when the global data was analysed, no gender differences were found. On the other hand, some authors have reported some gender differences; boys show a tendency to be more impulsive and have a poorer motor control than girls [37]. Our results, when analysed the scores in D2 Test of Attention, find also different behaviour patterns according to gender. Specifically, the improvement in D2 Scores is found to be better in girls than boys, according to our data.

To conclude, it is increasingly recognized that $\mathrm{ADHD}$ is a lifelong disorder and the focus on mental health interventions needs to be longterm: individualized plans that require ongoing evaluation, modification, and implementation, over months and years.

A key limitation of this study is the small number of participants. Taking this data into account, future research should focus also on the elaboration and assessment of intervention programs with a larger sample taking as a starting point the alteration in executive functions in children with ADHD.

\section{Conflicts of Interest}

The authors have declared that they have no competing or potential conflicts of interest. 


\section{References}

1. Epstein JN, Loren RE. Changes in the Definition of ADHD in DSM-5: Subtle but important. Neuropsychiatry 3(5), 455-458 (2013).

2. Stuss DT, Alexander MP. Is there a dysexecutive system? Philos. Trans. R. Soc. Lond. B. Biol. Sci 362(1481), 901-915 (2007).

3. Samango-Sprouse C. Frontal lobe development in childhood: The human frontal lobes: Functions and disorders, Guildford Press, New York (1999).

4. Barkley RA. Behavioral inhibition, sustained attention, and executive function: Constructing a unified theory of ADHD. Psychol. Bull 121(1), 65-94 (1997).

5. Castellanos FX, Tannock R. Neuroscience of attention-deficit/hyperactivity disorder: The search for endophenotypes. Nat. Rev. Neurosci 3(8), 617-628 (2002).

6. Pennington $\mathrm{BF}$, Ozonoff $\mathrm{S}$. Executive functions and developmental psychopathology. J. Child. Psychol. Psychiatry 37(1), 51-87 (1996).

7. Schachar R, Mota VL, Logan GD, et al. Confirmation of an inhibitory control deficit in attention-deficit/hyperactivity disorder. J. Abnorm. Child. Psychol 28(3), 227-235 (2000).

8. Thorell L, Eninger L, Brocki KC, et al. Childhood Executive Function Inventory (CHEXI): A promising measure for identifying young children with ADHD?.J. Clin. Exp. Neuropsychol 32(1), 38-43 (2010).

9. Fuster JM. The Prefrontal Cortex: Anatomy, Physiology and Neuropsychology of the Frontal Lobe, 2nd Edtn. Raven, New York (1997).

10. Stuss DT, Benson DF. The Frontal Lobes, Raven Press, New York (1986).

11. Krain AL, Castellanos FX. Brain development and ADHD. Clin. Psychol. Rev 26(4), 433-444 (2006).

12. Mackie $S$, Shaw $P$, Lenroot $R$, et al. Cerebellar development and clinical outcome in attention deficit hyperactivity disorder. Am. J. Psychiatry 164(4), 647-655 (2007).

13. Shaw P, Lerch J, Greenstein D, et al. Longitudinal mapping of cortical thickness and clinical outcome in children and adolescents with attention-deficit/ hyperactivity disorder. Arch. Gen. Psychiatry 63(5), 540-549 (2006).

14. Chafee MV, Goldman-Rakic PS. Matching patterns of activity in primate prefrontal area 8a and parietal area 7ip neurons during a spatial working memory task. J. Neurophysiol 79(6), 2919-2940 (1998).

15. Shaw P, Eckstrand K, Sharp W, et al. Attention-deficit/hyperactivity disorder is characterized by a delay in cortical maturation. Proc. Natl. Acad. Sci. USA 104(49), 19649-19654 (2007).

16. Casey BJ, Nigg JT, Durston S. New potential leads in the biology and treatment of attention deficit-hyperactivity disorder. Curr. Opin. Neurol 20(2), 119-124 (2007).

17. Sonuga-Barke E, Bitsakou P, Thompson M. Beyond the dual pathway model: Evidence for the dissociation of timing, inhibitory, and delay-related impairments in attentiondeficit/hyperactivity disorder. J. Am. Acad. Child. Adolesc. Psychiatry 49(4), 345-355 (2010).

18. Nigg JT, Casey BJ. An integrative theory of attention-deficit/hyperactivity disorder based on the cognitive and affective neurosciences. Dev. Psychopathol 17(3), 785-806 (2005).

19. Castellanos FX, Giedd JN, Marsh WL, et al. Quantitative brain magnetic resonance imaging in attention-deficit hyperactivity disorder. Arch. Gen. Psychiatry 53(7), 607616 (1996).

20. Carmona S, Vilarroya O, Bielsa A, et al. Global and regional gray matter reductions in ADHD: A voxelbased morphometric study. Neurosci. Lett 389 (2), 88-93 (2005).

21. Plessen KJ, Bansal R, Zhu H, et al. Hippocampus and amygdala morphology in attention-deficit/hyperactivity disorder. Arch. Gen. Psychiatry 63(7), 795-807 (2006).

22. Brickencamp R, Zilmer E. The d2 Test of Attention, Hogrefe \& Huber, Boston (1999).

23. Wilson BA, Emslie H, Evans JJ, et al. Behavioural Assessment of the Dysexecutive Syndrome (BADs), Pearson, London (1996).

24. Holmes J, Gathercole SE, Place M, et al. The Diagnostic Utility of Executive Function Assessments in the Identification of ADHD in Children. CAMH 15(1), 37-43 (2010).

25. Willcutt EG, Doyle AE, Nigg JT, et al. Validity of the executive function theory of attention-deficit/hyperactivity disorder: A meta-analytic review. Biol. Psychiatry 57(11), 1336-1346 (2005).

26. Toplak ME, Bucciarelli SM, Jain U, et al. Executive functions: performance-based measures and the behavior rating inventory of executive function (BRIEF) in adolescents with attention deficit/hyperactivity disorder (ADHD). Child. Neuropsychol 15(1), 53-72 (2009).

27. Brooks R. The self-esteem teacher: Seeds of selfesteem, Treehaus, New York (1991).

28. Setin J. Emotional self-regulation: A critical component of executive function: $L$. Meltzer (Edtn.) Guilford Press, New York (2010).

29. Tangney JP, Baumeister RF, Boone AL. High self-control predicts good adjustment, less pathology, better grades, and interpersonal success. J. Pers 72(2), 271-324 (2004).

30. Goldberg E. The executive brain: Frontal lobes and the civilized mind. Oxford University Press, New York (2001).

31. Gross JJ. Handbook of emotion regulation. Guilford Press, New York (2007).

32. Goleman D. Emotional intelligence. Bantam Books, New York (1995).

33. Sohlberg M, Mateer C. Cognitive Rehabilitation. An Integrative Neuropsychological Approach. Guilford Press, New York (2001).

34. Hooft I, Andersson K, Sejersen T, et al. Attention and memory training in children with acquired brain injuries. Acta. Paediatr 92(8), 935-40 (2003).

35. Dawson $P$, Guare R. Executive Skills in Children and Adolescents: a practical guide to assessment and intervention, The Guilford Press, Canada (2004).

36. Gomez R. Testing gender differential item functioning for ordinal and binary scored parent rated ADHD symptoms. Pers. Individ. Dif 42(4), 733-742 (2007).

37. Cole WR, Mostofsky SH, Larson JC, et al. Age-related changes in motor subtle signs among girls and boys with ADHD. Neurology 71(19), 1514-1520 (2008). 